\title{
Complexity growth rates for AdS black holes in massive gravity and $f(R)$ gravity
}

\author{
Wen-Di Guo ${ }^{\mathrm{a}}$, Shao-Wen Wei ${ }^{\mathrm{b}}$, Yan-Yan Lic ${ }^{\mathrm{c}}$, Yu-Xiao Liu ${ }^{\mathrm{d}}$ \\ Institute of Theoretical Physics, Lanzhou University, Lanzhou 730000, People's Republic of China
}

Received: 23 August 2017 / Accepted: 13 December 2017 / Published online: 26 December 2017

(C) The Author(s) 2017. This article is an open access publication

\begin{abstract}
The "complexity = action" duality states that the quantum complexity is equal to the action of the stationary AdS black hole within the Wheeler-DeWitt patch at late time approximation. We compute the action growth rates of the neutral and charged black holes in massive gravity and the neutral, charged and Kerr-Newman black holes in $f(R)$ gravity to test this conjecture. Besides, we investigate the effects of the massive graviton terms, higher derivative terms and the topology of the black hole horizon on the complexity growth rate.
\end{abstract}

\section{Introduction}

The quantum computational complexity was recently proposed by Susskind et al. [1-5] for the first time. They connected the black hole interior with the quantum computational complexity, which was defined as the minimal number of elementary operations (also named quantum gates) on constructing the boundary quantum state from a reference quantum state $[4,5]$. This is a remarkable progress on understanding black hole interior. After that, the quantum complexity has been extensively investigated [6-29]. The gravitational action with null boundaries was proposed and discussed in detail in Ref. [8], and the connection between the second law of thermodynamics with the quantum complexity was investigated in Ref. [19].

Stanford and Susskind first related the complexity $\mathcal{C}$ with the spatial volume $V$ of the Einstein-Rosen bridge which connects two boundaries as [30]

$\mathcal{C} \sim \frac{V}{G L}$,

\footnotetext{
a e-mail: guowd14@1zu.edu.cn

be-mail: weishw@1zu.edu.cn

c e-mail: liyy2015@1zu.edu.cn

de-mail: liuyx@1zu.edu.cn
}

where $G$ is the Newton constant and $L$ is a length scale that should be chosen to be either the Anti-de Sitter (AdS) radius or the radius of the black hole horizon. This conjecture, named Complexity-Volume (CV) duality, has a lot of nice features and it has been tested in the shock wave geometries [5]. It is worthwhile noting that the length scale $L$ should be chosen according to the explicit situation. The subsequent Complexity-Action (CA) duality does not involve any ambiguous quantities and preserves all the good features of the CV duality [5]. The CA duality relates the quantum complexity to the action of the black hole in the Wheeler-DeWitt (WDW) patch:

$\mathcal{C}=\frac{A}{\pi \hbar}$

The authors of Ref. [15] revisited the connection between the thermodynamical volume of the black hole and the action growth rate in the WDW patch at late time approximation. The UV divergence of the action in that region was investigated in Refs. [10,17]. It was pointed out by Lloyd that the quantum complexity growth rate is bounded by [31]

$\frac{\mathrm{d} C}{\mathrm{~d} t} \leq \frac{2 E}{\pi \hbar}$,

where $E$ is the average energy of the quantum state relating to the ground state. If the CA duality is correct, the action growth rates of the black holes in the WDW patch will also be bounded. This was tested by computing the action growth rate. And some concrete forms of the action growth rate of the AdS black holes in the WDW patch at the late time approximation were proposed $[4,5]$.

However, the authors of Ref. [32] found that the above bound will be violated for both small and large charged static 
AdS black holes, so they proposed a new bound

$\frac{\mathrm{d} A}{\mathrm{~d} t} \leq\left(M-\Omega_{+} J-\mu_{+} Q\right)-\left(M-\Omega_{-} J-\mu_{-} Q\right)$,

where $\Omega$ and $\mu$ denote, respectively, the angular velocity and chemical potential of the black holes, and the parameters $M, J$, and $Q$ are the black hole mass, angular momentum, and charge, respectively. The subscript $+(-)$ denotes the quantities evaluated at the outer (inner) horizon. They conjectured that all stationary AdS black holes could saturate this bound for gravity theories without higher derivative terms of curvature. As a test, the action growth rates of $D$ dimensional Reissner-Nordström-AdS black holes, KerrAdS black holes, rotating and charged BTZ black holes in general relativity (GR), as well as the charged GaussBonnet-AdS black holes have been calculated. This conjecture was generalized to a more compact form in Ref. [20]. It was shown that the difference of the generalized enthalpy between the two corresponding horizons decides the action growth rate [20] and strong energy conditions of the matter outside the horizon of the black holes can ensure the validity of the complexity growth rate bound [18]. The action growth rates of the charged black holes with a single horizon were also studied in Ref. [24]. The Lloyd bounds of the action growth rates of black holes in Born-Infeld gravity and in $f(R)$ gravity were also tested in Refs. [22,23] by considering the contributions from the null surface and the joints. They found that the Lloyd bounds for charged black holes will usually be violated in both Born-Infeld gravity and $f(R)$ gravity. Besides, the action growth rates based on the CA conjecture were also studied in massive gravity and higher derivative theories in Refs. [33,34]. In this paper, we only focus on the conjecture (4) rather than the Lloyd bound, because the conjecture (4) is based on the violation of the Lloyd bound.

This paper is organized as follows. In Sect. 2, we first verify the KN case in GR. In Sect. 3 we calculate the action growth rate of the neutral and charged black holes in higher dimensional massive gravity within the WDW patch at the late time approximation, and study the influence of the massive graviton terms. Besides, whether the parameter $k$, which denotes the topology of the black hole horizon, will affect the action growth rate is studied. In Sect. 4 the action growth rate of the black holes in $f(R)$ gravity is studied, for the cases of neutral, charged, and KN black holes. Finally, the conclusions are given in Sect. 5.

\section{Action growth rate of Kerr-Newman black holes in GR}

Before dealing with massive gravity and $f(R)$ gravity, we start with the KN black holes in GR. The action is given by

$$
\begin{aligned}
\mathcal{A}= & \mathcal{A}_{\mathrm{bk}}+\mathcal{A}_{\mathrm{bd}} \\
= & \frac{1}{16 \pi G} \int_{M} \mathrm{~d}^{4} x \sqrt{-g}\left(R-2 \Lambda-G F_{\mu \nu} F^{\mu \nu}\right) \\
& +\frac{1}{8 \pi G} \int_{\partial M} \mathrm{~d}^{3} x \sqrt{-h} K
\end{aligned}
$$

where $\mathcal{A}_{\mathrm{bk}}$ and $\mathcal{A}_{\mathrm{bd}}$ denote the actions of the bulk and boundary terms, respectively, and $h$ is the induced metric of the hypersurface and $K$ is the trace of the extrinsic curvature. The solutions of the metric and the electric potential are given in Refs. [35,36]:

$$
\begin{aligned}
\mathrm{d} s^{2}= & -\left(\frac{\triangle_{r}}{\rho^{2}}-\frac{\triangle_{\theta} \sin ^{2} \theta}{\rho^{2}} a^{2}\right) \mathrm{d} t^{2}+\frac{\rho^{2}}{\triangle_{r}} \mathrm{~d} r^{2}+\frac{\rho^{2}}{\triangle_{\theta}} \mathrm{d} \theta^{2} \\
& +2 \frac{a \triangle_{r} \sin ^{2} \theta-a\left(r^{2}+a^{2}\right) \triangle_{\theta} \sin ^{2} \theta}{\rho^{2} \Xi} \mathrm{d} t \mathrm{~d} \phi \\
& +\frac{\sin ^{2} \theta}{\rho^{2} \Xi^{2}}\left[\left(r^{2}+a^{2}\right)^{2} \triangle_{\theta}-a^{2} \triangle_{r} \sin ^{2} \theta\right] \mathrm{d} \phi^{2}, \\
A= & -\frac{q r}{\rho^{2}} \mathrm{~d} t+\frac{q r a \sin ^{2} \theta}{\Xi \rho^{2}} \mathrm{~d} \phi .
\end{aligned}
$$

Here we adopt the conventions of Ref. [37]. The metric functions are given by

$$
\begin{aligned}
& \triangle_{r}=\left(a^{2}+r^{2}\right)\left(1+\frac{r^{2}}{l^{2}}\right)-2 G m r+G q^{2}, \\
& \triangle_{\theta}=1-\frac{a^{2} \cos ^{2} \theta}{l^{2}}, \quad \rho^{2}=a^{2} \cos ^{2} \theta+r^{2}, \\
& \Xi=1-\frac{a^{2}}{l^{2}},
\end{aligned}
$$

where $a$ is the rotational parameter. Note that this solution is valid only for $a^{2}<l^{2}$. The thermodynamical quantities are [37]

$$
\begin{aligned}
& M=\frac{m}{\Xi^{2}}, J=\frac{a m}{\Xi^{2}}, Q=\frac{q}{\Xi} \\
& \mu_{ \pm}=\frac{q r_{ \pm}}{\left(r_{ \pm}^{2}+a^{2}\right)}, \Omega_{ \pm}=\frac{a \Xi}{r_{ \pm}^{2}+a^{2}} .
\end{aligned}
$$

Solving $\triangle_{r}\left(r_{ \pm}\right)=0$, we can get

$$
\begin{aligned}
m & =\frac{\left(r_{-}+r_{+}\right)\left(a^{2}+l^{2}+r_{-}^{2}+r_{+}^{2}\right)}{2 G l^{2}}, \\
q^{2} & =\frac{r_{-} r_{+}\left(l^{2}+r_{-}^{2}+r_{+}^{2}+r_{-} r_{+}\right)}{G l^{2}} \\
& +a^{2} \frac{\left(r_{-} r_{+}-l^{2}\right)}{G l^{2}} .
\end{aligned}
$$

The determinant of this metric is

$$
\sqrt{-g}=\frac{\sin \theta}{\Xi} \rho^{2}
$$


The Ricci scalar $R$ and the field strength $F^{2}$ can be calculated straightforwardly as

$$
\begin{aligned}
R= & -\frac{12}{l^{2}} \\
F^{2}= & -4 q^{2}\left(\frac{a^{4} \cos (4 \theta)+3 a^{4}+4 a^{2}\left(a^{2}-6 r^{2}\right) \cos (2 \theta)}{\left(a^{2} \cos (2 \theta)+a^{2}+2 r^{2}\right)^{4}}\right. \\
& \left.+\frac{8 r^{4}-24 a^{2} r^{2}}{\left(a^{2} \cos (2 \theta)+a^{2}+2 r^{2}\right)^{4}}\right)
\end{aligned}
$$

Then we can get the action growth rate of the EinsteinMaxwell part

$$
\begin{aligned}
\frac{\mathrm{d} \mathcal{A}_{\mathrm{bk}}}{\mathrm{d} t}= & \frac{2 \pi}{16 \pi G} \int_{r_{-}}^{r_{+}} \int_{0}^{\pi} \sqrt{-g}\left(R-2 \Lambda-G F_{\mu \nu} F^{\mu \nu}\right) \mathrm{d} \theta \mathrm{d} r \\
= & \frac{1}{2}\left(r_{+}-r_{-}\right)\left(\frac{l^{2} q^{2}\left(a^{2}-r_{-} r_{+}\right)}{\left(a^{2}-l^{2}\right)\left(a^{2}+r_{-}^{2}\right)\left(a^{2}+r_{+}^{2}\right)}\right. \\
& \left.+\frac{a^{2}+r_{-}^{2}+r_{-} r_{+}+r_{+}^{2}}{G\left(a^{2}-l^{2}\right)}\right) .
\end{aligned}
$$

Note that we integrate the action from the inner horizon $r_{-}$ to the outer horizon $r_{+}$because other regions of the WDW patch do not contribute to the action growth rate at late time approximation. The contribution of the region outside the horizon is independent on time and that of the region behind the past horizon shrinks exponentially to zero [5]. The trace of the extrinsic curvature $K$ can be gotten as

$$
\begin{aligned}
K & =\frac{1}{\sqrt{-g}} \partial_{r}\left(\frac{\sqrt{-g}}{\rho} \sqrt{\triangle_{r}}\right) \\
& =\frac{r \sqrt{\triangle_{r}}}{\rho^{3}}+\frac{\partial_{r} \triangle_{r}}{2 \rho \sqrt{\triangle_{r}}} .
\end{aligned}
$$

So the contribution from the boundary term will be

$$
\begin{aligned}
\frac{\mathrm{d} \mathcal{A}_{\mathrm{bd}}}{\mathrm{d} t} & =\left.\frac{\partial_{r} \Delta r}{4 G \Xi}\right|_{r_{-}} ^{r_{+}} \\
& =\left(r_{+}-r_{-}\right) \frac{a^{2}+l^{2}+2\left(r_{-}^{2}+r_{+} r_{-}+r_{+}^{2}\right)}{2 G\left(l^{2}-a^{2}\right)},
\end{aligned}
$$

where we have used $\triangle_{r}\left(r_{ \pm}\right)=0$, and the angle part has been integrated. In the end, we can get the action growth rate of a KN AdS black hole in GR

$$
\begin{aligned}
\frac{\mathrm{d} \mathcal{A}}{\mathrm{d} t}= & \left(r_{+}-r_{-}\right)\left(\frac{a^{4}\left(2 l^{2}+r_{-}^{2}+r_{+}^{2}\right)}{2 G\left(l^{2}-a^{2}\right)\left(a^{2}+r_{-}^{2}\right)\left(a^{2}+r_{+}^{2}\right)}\right. \\
& +\frac{a^{2}\left(l^{2}\left(r_{-}-r_{+}\right)^{2}+\left(r_{-}^{2}+r_{+}^{2}\right)^{2}\right)}{2 G\left(l^{2}-a^{2}\right)\left(a^{2}+r_{-}^{2}\right)\left(a^{2}+r_{+}^{2}\right)} \\
& \left.+\frac{2 r_{-}^{2} r_{+}^{2}\left(l^{2}+r_{-}^{2}+r_{+}^{2}+r_{-} r_{+}\right)}{2 G\left(l^{2}-a^{2}\right)\left(a^{2}+r_{-}^{2}\right)\left(a^{2}+r_{+}^{2}\right)}\right) .
\end{aligned}
$$

With the help of the thermodynamic quantities (9), we can rewrite it in the following form:

$\frac{\mathrm{d} \mathcal{A}}{\mathrm{d} t}=\left(M-\Omega_{+} J-\mu_{+} Q\right)-\left(M-\Omega_{-} J-\mu_{-} Q\right)$.

Clearly, this is just the action growth rate of a KN AdS black hole in GR speculated in Ref. [32].

\section{Action growth rate of black holes in massive gravity}

We first give a brief review of the black hole solutions in $(n+2)$-dimensional massive gravity [38-42]. The action is given by $[43,44]$

$$
\begin{aligned}
\mathcal{A}= & \mathcal{A}_{\mathrm{bk}}+\mathcal{A}_{\mathrm{bd}} \\
= & \frac{1}{16 \pi G} \int_{M} \mathrm{~d}^{n+2} x \sqrt{-g}\left(R+\frac{(n+1) n}{l^{2}}-G F^{2}\right. \\
& \left.+m^{2} \sum_{i=1}^{4} c_{i} \mathcal{U}_{i}(g, \hat{f})\right)+\frac{1}{8 \pi G} \int_{\partial M} \mathrm{~d}^{n+1} x \sqrt{-h} K,
\end{aligned}
$$

where $g, \hat{f}$, and $m$ are, respectively, the spacetime metric, the reference metric, and the mass of the graviton. The coefficients $c_{i}$ are negative constants if one requires $m^{2}>0$ [44], and $\mathcal{U}_{i}$ are defined by the following symmetric polynomials:

$\mathcal{U}_{1}=[\mathcal{K}]$,

$\mathcal{U}_{2}=[\mathcal{K}]^{2}-\left[\mathcal{K}^{2}\right]$,

$\mathcal{U}_{3}=[\mathcal{K}]^{3}-3[\mathcal{K}]\left[\mathcal{K}^{2}\right]+2\left[\mathcal{K}^{3}\right]$,

$\mathcal{U}_{4}=[\mathcal{K}]^{4}-6\left[\mathcal{K}^{2}\right][\mathcal{K}]^{2}+8\left[\mathcal{K}^{3}\right][\mathcal{K}]+3\left[\mathcal{K}^{2}\right]^{2}-6\left[\mathcal{K}^{4}\right]$,

where $[X]$ denotes the trace of the matrix $X$. The matrix $\mathcal{K}^{\mu}{ }_{v}$ is defined as $\mathcal{K}_{v}^{\mu}=\sqrt{g^{\mu \alpha} \hat{f}_{\alpha \nu}}$. Varying action (18) with respect to the metric $g_{\mu \nu}$ and the gauge field $A_{\mu}$ we can get the field equations:

$$
\begin{aligned}
& R_{\mu \nu}-\frac{1}{2} R g_{\mu \nu}-\frac{1}{2 l^{2}} n(n+1) g_{\mu \nu}+m^{2} \chi_{\mu \nu} \\
& =2 G\left(F_{\mu \sigma} F_{\nu}^{\sigma}-\frac{1}{4} g_{\mu \nu} F^{2}\right), \\
& \nabla_{\mu} F^{\mu \nu}=0,
\end{aligned}
$$

where the mass term $\chi_{\mu \nu}$ is given by

$$
\begin{aligned}
\chi_{\mu \nu}=- & \frac{c_{1}}{2}\left(\mathcal{U}_{1} g_{\mu \nu}-\mathcal{K}_{\mu \nu}\right) \\
& -\frac{c_{2}}{2}\left(\mathcal{U}_{2} g_{\mu \nu}-2 \mathcal{U}_{1} \mathcal{K}_{\mu \nu}+2 \mathcal{K}_{\mu \nu}^{2}\right) \\
& -\frac{c_{3}}{2}\left(\mathcal{U}_{3} g_{\mu \nu}-3 \mathcal{U}_{2} \mathcal{K}_{\mu \nu}+6 \mathcal{U}_{1} \mathcal{K}_{\mu \nu}^{2}-6 \mathcal{K}_{\mu \nu}^{3}\right) \\
& -\frac{c_{4}}{2}\left(\mathcal{U}_{4} g_{\mu \nu}-4 \mathcal{U}_{3} \mathcal{K}_{\mu \nu}+12 \mathcal{U}_{2} \mathcal{K}_{\mu \nu}^{2}\right. \\
& \left.-24 \mathcal{U}_{1} \mathcal{K}_{\mu \nu}^{3}+24 \mathcal{K}_{\mu \nu}^{4}\right) .
\end{aligned}
$$


The solution for a static black hole is [44]:

$$
\begin{aligned}
\mathrm{d} s^{2}= & -f(r) \mathrm{d} t^{2}+f^{-1}(r) \mathrm{d} r^{2}+r^{2} \hat{h}_{i j} \mathrm{~d} x^{i} \mathrm{~d} x^{j} \\
& (i, j=1,2,3, \cdots, n), \\
\hat{f}_{\mu \nu}= & \operatorname{diag}\left(0,0, \hat{h}_{i j}\right), \\
A_{\mu}= & \left(A_{t}, 0,0, \cdots, 0\right),
\end{aligned}
$$

where $\hat{h}_{i j}$ is the induced metric of the hypersurface space. The explicit forms of $f(r)$ and $A_{t}$ are [44]

$$
\begin{aligned}
f(r)= & k+\frac{r^{2}}{l^{2}}-\frac{2 G m_{0}}{r^{n-1}}+\frac{2 G q^{2}}{n(n-1) r^{2(n-1)}} \\
& +\frac{c_{1} m^{2}}{n} r+c_{2} m^{2}+\frac{(n-1) c_{3} m^{2}}{r} \\
& +\frac{(n-1)(n-2) c_{4} m^{2}}{r^{2}}, \\
A_{t}= & \frac{q}{(n-1) r^{n-1}}-\frac{q}{(n-1) r_{+}^{n-1}},
\end{aligned}
$$

where $k=-1,0,1$ denotes the topology of the black hole horizon, corresponding to hyperbolic, Ricci flat, or spherical, respectively. The physical mass $M$ and charge $Q$ of the black hole are given by

$$
\begin{aligned}
M & =\frac{n V_{n}}{8 \pi} m_{0}, \\
Q & =\frac{V_{n}}{4 \pi} q,
\end{aligned}
$$

where $V_{n}$ is the volume of the $n$-dimensional space that depends on the topology of the black hole horizon. The $P-V$ criticality and the Van der Waals like behavior of the massive gravity black holes have been investigated in Refs. [45-52].

Next we will investigate the effects of the mass of the graviton, the dimension of spacetime, and the topology of the black hole horizon on the action growth rates for both the neutral and the charged massive black holes.

\subsection{Action growth rate of the neutral black holes in higher} dimensional massive gravity

For neutral black holes, there is no Maxwell field part in the action. With the metric (22), we can calculate the Ricci scalar $R$ for the neutral black holes:

$$
\begin{aligned}
R= & -\frac{(n+1)(n+2)}{l^{2}}-\frac{c_{1} m^{2}(n+1)}{r}-\frac{c_{2} m^{2} n(n-1)}{r^{2}} \\
& -\frac{c_{3} m^{2}(n-1)^{2}(n-2)}{r^{3}} \\
& -\frac{c_{4} m^{2}(n-1)(n-2)^{2}(n-3)}{r^{4}}
\end{aligned}
$$

Following Refs. [5,32], the action growth rate of the bulk action in the WDW patch in the late time approximation limit is

$$
\begin{aligned}
\frac{\mathrm{d} \mathcal{A}_{\mathrm{bk}}}{\mathrm{d} t}= & \frac{V_{n}}{16 \pi G} \int_{0}^{r_{h}} r^{n}\left(R+\frac{(n+1) n}{l^{2}}\right. \\
& \left.+m^{2} \sum_{i=1}^{4} c_{i} \mathcal{U}_{i}(g, f)\right) \mathrm{d} r \\
= & \frac{V_{n}}{16 \pi G}\left(-m^{2} \frac{c_{1}}{n} r_{h}^{n}+m^{2} c_{3}(n-1) r_{h}^{n-2}\right. \\
& \left.+2 m^{2} c_{4}(n-1)(n-2) r_{h}^{n-3}-\frac{2}{l^{2}} r^{n+1}\right),
\end{aligned}
$$

where $r_{h}$ denotes the horizon radius. Next, let us turn to the YGH boundary term. The trace of the extrinsic curvature with the metric (22) is

$$
\begin{aligned}
K & =\frac{1}{r^{n}} \partial_{r}\left(r^{n} \sqrt{f}\right) \\
& =\frac{n}{r} \sqrt{f}+\frac{f^{\prime}}{2 \sqrt{f}},
\end{aligned}
$$

where the prime denotes the derivative with respect to $r$. So the action growth rate of the surface term in the WDW patch is

$$
\begin{aligned}
\frac{\mathrm{d} \mathcal{A}_{\mathrm{bd}}}{\mathrm{d} t}= & \frac{V_{n}}{8 \pi G}\left[r^{n} \sqrt{f}\left(\frac{n}{r} \sqrt{f}+\frac{f^{\prime}}{2 \sqrt{f}}\right)\right]_{0}^{r_{h}} \\
= & \frac{V_{n}}{8 \pi G}\left(n r_{h}^{n-1} k+\frac{n+1}{l^{2}} r_{h}^{n+1}\right. \\
& +\left(1+\frac{1}{2 n}\right) c_{1} m^{2} r_{h}^{n}+n c_{2} m^{2} r_{h}^{n-1} \\
& +\left(n-\frac{1}{2}\right)(n-1) c_{3} m^{2} r_{h}^{n-2} \\
& \left.+(n-1)^{2}(n-2) c_{4} m^{2} r_{h}^{n-3}\right) .
\end{aligned}
$$

Then we can get the total action growth rate of the higher dimensional massive neutral black holes in the WDW patch in the late time approximation limit:

$$
\begin{aligned}
\frac{\mathrm{d} \mathcal{A}}{\mathrm{d} t}= & \frac{\mathrm{d} \mathcal{A}_{\mathrm{bk}}}{\mathrm{d} t}+\frac{\mathrm{d} \mathcal{A}_{\mathrm{bd}}}{\mathrm{d} t} \\
= & \frac{n V_{n}}{8 \pi G}\left(k r_{h}^{n-1}+\frac{r_{h}^{n+1}}{l^{2}}+\frac{1}{n} c_{1} m^{2} r_{h}^{n}\right. \\
& +c_{2} m^{2} r_{h}^{n-1}+(n-1) c_{3} m^{2} r_{h}^{n-2} \\
& \left.+(n-1)(n-2) c_{4} m^{2} r_{h}^{n-3}\right) .
\end{aligned}
$$

Solving $f\left(r_{h}\right)=0$, we get

$$
\begin{aligned}
m_{0}= & \frac{1}{2 G}\left(k r_{h}^{n-1}+\frac{r_{h}^{n+1}}{l^{2}}+\frac{1}{n} c_{1} m^{2} r_{h}^{n}\right. \\
& +c_{2} m^{2} r_{h}^{n-1}+(n-1) c_{3} m^{2} r_{h}^{n-2} \\
& \left.+(n-1)(n-2) c_{4} m^{2} r_{h}^{n-3}\right) .
\end{aligned}
$$


Combining with Eq. (27), we can get the same result as Refs. [32,33]:

$\frac{\mathrm{d} \mathcal{A}}{\mathrm{d} t}=2 M$.

If the CA conjecture is correct, then we will get

$$
\frac{\mathrm{d} \mathcal{C}}{\mathrm{d} t}=\frac{2 M}{\pi \hbar} .
$$

The result is independent of the spacetime dimension, the mass of graviton, and the topology of the black hole. If a neutral stationary AdS black hole in massive gravity has the same mass as the black hole in GR, they will have the same growth rate of complexity. So we conclude that a neutral massive black hole will also be the fastest computer.

\subsection{Action growth rate of the charged black holes in higher dimensional massive gravity}

For the case of a charged black hole, the field strength of the electromagnetic field turns out to be

$F^{2}=-2 q^{2} r^{-2 n}$

and the parameters $m_{0}$ and $q$ can be solved from $f\left(r_{ \pm}\right)=0$,

$$
\begin{aligned}
m_{0}= & \frac{r_{+} r_{-}^{2 n-1}}{2 G\left(r_{+} r_{-}^{n}-r_{-} r_{+}^{n}\right)}\left(k+\frac{r_{-}^{2}}{l^{2}}+\frac{c_{1}}{n} m^{2} r_{-}+c_{2} m^{2}\right. \\
& \left.+(n-1) c_{3} m^{2} \frac{1}{r_{-}}+(n-1)(n-2) c_{4} m^{2} \frac{1}{r_{-}^{2}}\right) \\
& -\frac{r_{+}^{2 n-1} r_{-}}{2 G\left(r_{+} r_{-}^{n}-r_{-} r_{+}^{n}\right)}\left(k+\frac{r_{+}^{2}}{l^{2}}+\frac{c_{1}}{n} m^{2} r_{+}+c_{2} m^{2}\right. \\
& \left.+(n-1) c_{3} m^{2} \frac{1}{r_{+}}+(n-1)(n-2) c_{4} m^{2} \frac{1}{r_{+}^{2}}\right) \\
q^{2}= & \frac{(n-1)}{2 G}\left(n k r_{-}^{n-1} r_{+}^{n-1}+\frac{n\left(r_{-}^{2 n} r_{+}^{n-1}-r_{-}^{n-1} r_{+}^{2 n}\right)}{l^{2}\left(r_{-}^{n-1}-r_{+}^{n-1}\right)}\right. \\
& -\frac{c_{1} m^{2}\left(r_{-}^{n}-r_{+}^{n}\right)}{r_{-}^{1-n}-r_{+}^{1-n}+n c_{2} m^{2} r_{+}^{n-1} r_{-}^{n-1}} \\
& +\frac{n c_{3} m^{2}\left(r_{-}^{n-2}-r_{+}^{n-2}\right)}{r_{+}^{1-n}-r_{-}^{1-n}} \\
& \left.+\frac{(n-1)(n-2) c_{4} m^{2}\left(r_{-}^{n-3}-r_{+}^{n-3}\right)}{r_{+}^{1-n}-r_{-}^{1-n}}\right) .
\end{aligned}
$$

The bulk action growth rate of the charged massive black hole within the WDW patch in the late time approximation limit can be calculated straightforwardly:

$$
\begin{aligned}
\frac{\mathrm{d} \mathcal{A}_{\mathrm{bk}}}{\mathrm{d} t}= & \frac{V_{n}}{8 \pi G}\left[k\left(r_{+}^{n-1}-r_{-}^{n-1}\right)+\frac{c_{1}}{2 n} m^{2}\left(r_{+}^{n}-r_{-}^{n}\right)\right. \\
& +c_{2} m^{2}\left(r_{+}^{n-1}-r_{-}^{n-1}\right) \\
& +\frac{3(n-1) c_{3}}{2} m^{2}\left(r_{+}^{n-2}-r_{-}^{n-2}\right) \\
& \left.+2 c_{4} m^{2}(n-2)(n-1)\left(r_{+}^{n-3}-r_{-}^{n-3}\right)\right] .
\end{aligned}
$$

Besides, the contribution from the YGH surface term within the WDW patch is

$$
\begin{aligned}
\frac{\mathrm{d} \mathcal{A}_{\mathrm{bd}}}{\mathrm{d} t}= & \frac{V_{n}}{16 \pi G}\left[2 k(n-1)\left(r_{+}^{n-1}-r_{-}^{n-1}\right)\right. \\
& +c_{1} m^{2} \frac{(2 n-1)}{n}\left(r_{+}^{n}-r_{-}^{n}\right) \\
& +2 c_{2} m^{2}(n-1)\left(r_{+}^{n-1}-r_{-}^{n-1}\right) \\
& +c_{3} m^{2}(n-1)(2 n-3)\left(r_{+}^{n-2}-r_{-}^{n-2}\right) \\
& \left.+2 c_{4} m^{2}(n-2)^{2}(n-1)\left(r_{+}^{n-3}-r_{-}^{n-3}\right)\right] .
\end{aligned}
$$

Therefore, the total action growth rate of the charged massive black holes in the WDW patch in the late time approximation limit is

$$
\begin{aligned}
\frac{\mathrm{d} \mathcal{A}}{\mathrm{d} t} & =\frac{\mathrm{d} \mathcal{A}_{\mathrm{bk}}}{\mathrm{d} t}+\frac{\mathrm{d} \mathcal{A}_{\mathrm{bd}}}{\mathrm{d} t} \\
& =\frac{4 \pi}{(n-1) V_{n}} Q^{2}\left(\frac{1}{r_{-}^{n-1}}-\frac{1}{r_{+}^{n-1}}\right) \\
& =\mu_{-} Q-\mu_{+} Q,
\end{aligned}
$$

where we have used the charge of the black hole (28), and the chemical potential is defined as follows:

$\mu_{ \pm}=\frac{q}{(n-1) r_{ \pm}^{n-1}}$.

From Eq. (41), we can see that, no matter what value $k$ takes, the result is consistent with that of Ref. [32]. In other words, the topology of the charged black hole horizon will not affect the quantum complexity growth rate. However, as the authors of [33] showed, when the mass $M$ and charge $Q$ of the massive gravity black holes are the same as that of the black holes in GR, the complexity of the charged black holes in massive gravity theory will grow faster than that of the charged black holes in GR. The reason is as follows. If we define the quantities in GR with a hat, then we will get

$$
\begin{aligned}
\hat{f}(r)-f(r)= & -m^{2}\left(\frac{c_{1}}{n} r+c_{2}+\frac{(n-1) c_{3}}{r}\right. \\
& \left.+\frac{(n-1)(n-2) c_{4}}{r^{2}}\right)>0,
\end{aligned}
$$

for negative parameters $c_{i}$, which will ensure the positivity of the mass square of graviton. So we have the following relations: 
$r_{-}<\hat{r}_{-}$and $r_{+}>\hat{r}_{+}$.

Having

$\frac{\mathrm{d} \mathcal{C}}{\mathrm{d} t}=\frac{2 \kappa^{2}}{V_{n}} Q^{2}\left(\frac{1}{r_{-}^{n-1}}-\frac{1}{r_{+}^{n-1}}\right)$,

and

$\frac{\mathrm{d} \hat{\mathcal{C}}}{\mathrm{d} t}=\frac{2 \kappa^{2}}{V_{n}} Q^{2}\left(\frac{1}{\hat{r}_{-}^{n-1}}-\frac{1}{\hat{r}_{+}^{n-1}}\right)$,

one has

$\frac{\mathrm{d} \mathcal{C}}{\mathrm{d} t}>\frac{\mathrm{d} \hat{\mathcal{C}}}{\mathrm{d} t}$

So, charged black holes in massive gravity are faster computers than charged black holes in GR, though the bound (4) is not exceeded.

\section{Action growth rate of black holes in $f(R)$ gravity}

The action for $f(R)$-Maxwell gravity is

$$
\begin{aligned}
\mathcal{A}= & \mathcal{A}_{\mathrm{bk}}+\mathcal{A}_{\mathrm{bd}} \\
= & \frac{1}{16 \pi G} \int_{M} \mathrm{~d}^{4} x \sqrt{-g}\left[R+f(R)-G F^{2}\right] \\
& +\frac{1}{8 \pi G} \int_{\partial M} \mathrm{~d}^{3} x \sqrt{-h}\left[\left(1+f^{\prime}(R)\right) K\right],
\end{aligned}
$$

where the prime denotes the derivative with respect to $R$, and the YGH surface term is different from that of in GR [53]. The static black hole solution was found in Ref. [54]. The authors assumed the following metric form:

$\mathrm{d} s^{2}=-N(r) \mathrm{d} t^{2}+\frac{\mathrm{d} r^{2}}{N(r)}+r^{2} \mathrm{~d} \Omega_{2}^{2}$,

with the metric function given by

$N(r)=1-\frac{2 G m}{r}+\frac{G q^{2}}{\left(1+f^{\prime}\left(R_{0}\right)\right) r^{2}}-\frac{R_{0}}{12} r^{2}$.

Here the parameters $m$ and $q$ are related to the physical mass and charge,

$$
\begin{aligned}
M & =\left(1+f^{\prime}\left(R_{0}\right)\right) m, \\
Q & =\frac{q}{\sqrt{1+f^{\prime}\left(R_{0}\right)}} .
\end{aligned}
$$

According to Ref. [55], $R_{0}$ is the constant scalar curvature

$R_{0}=\frac{2 f\left(R_{0}\right)}{f^{\prime}\left(R_{0}\right)-1}=4 \Lambda=-\frac{12}{l^{2}}$,

where $\Lambda$ is the cosmology constant and $l$ is the AdS radius. Note that, in order to get constant curvature, the trace of the energy-momentum tensor of the Maxwell field should be zero, in other words, the spacetime should be four dimensional [55]. The other solutions and thermodynamical qualities for $f(R)$ gravity black holes have also been investigated in Refs. [56-65]. The authors of [32] conjectured that only the stationary black holes in gravity theories without higher derivative terms of curvature can saturate Eq. (4). So what will the situation be in $f(R)$ gravity?

\subsection{Action growth rate of the neutral black holes in $f(R)$ gravity}

In this section we will calculate the action growth rate of a static neutral black hole in $f(R)$ gravity within the WDW patch in the late time approximation limit.

We can get the mass parameter from $N\left(r_{h}\right)=0$ :

$m=\frac{1}{2 G}\left(r_{h}+\frac{r_{h}^{3}}{l^{2}}\right)$.

The contribution from the bulk action is

$$
\begin{aligned}
\frac{\mathrm{d} \mathcal{A}_{b k}}{\mathrm{~d} t} & =\frac{4 \pi}{16 \pi G} \int_{0}^{r_{h}} r^{2}\left(f\left(R_{0}\right)+R_{0}\right) \mathrm{d} r \\
& =-\frac{1+f^{\prime}\left(R_{0}\right)}{2 G l^{2}} r_{h}^{2} .
\end{aligned}
$$

The extrinsic curvature with the metric (49) is

$K=\frac{2}{r} \sqrt{N}+\frac{N^{\prime}}{2 \sqrt{N}}$

so the action growth rate of the YGH surface term in the WDW patch in the late time approximation limit is

$$
\begin{aligned}
\frac{\mathrm{d} \mathcal{A}_{\mathrm{bd}}}{\mathrm{d} t} & =\left.\frac{4 \pi}{8 \pi G} r^{2}\left(1+f^{\prime}\left(R_{0}\right)\right) \sqrt{N} K\right|_{0} ^{r_{h}} \\
& =\frac{1+f^{\prime}\left(R_{0}\right)}{2 G}\left(2 r_{h}+\frac{3}{l^{2}} r_{h}^{3}\right) .
\end{aligned}
$$

Thus the total action growth rate will be

$$
\begin{aligned}
\frac{\mathrm{d} \mathcal{A}}{\mathrm{d} t} & =\frac{\mathrm{d} \mathcal{A}_{\mathrm{bk}}}{\mathrm{d} t}+\frac{\mathrm{d} \mathcal{A}_{\mathrm{bd}}}{\mathrm{d} t} \\
& =2\left(1+f^{\prime}\left(R_{0}\right)\right) m \\
& =2 M,
\end{aligned}
$$

where we have used Eqs. (51) and (53). The interesting thing is that, in $f(R)$ gravity theory, the action growth rate Eq. (4) can also be saturated. That is to say, the higher derivative term of curvature in this theory does not change the action growth rate, so the neutral black holes in $f(R)$ gravity will still be the fastest computer. This result has been observed in Refs. $[23,34]$. 
4.2 Action growth rate of charged black holes in $f(R)$ gravity

The electromagnetic field strength is

$F^{2}=-\frac{2 q^{2}}{r^{4}}$.

The parameters of the mass and charge in the function $N(r)$ can be solved from $N\left(r_{ \pm}\right)=0$ :

$$
\begin{aligned}
m & =\frac{\left(r_{-}+r_{+}\right)\left(l^{2}+r_{-}^{2}+r_{+}^{2}\right)}{2 G l^{2}} \\
q^{2} & =\frac{r_{-} r_{+}\left(1+f^{\prime}\left(R_{0}\right)\right)\left(l^{2}+r_{-}^{2}+r_{+}^{2}+r_{-} r_{+}\right)}{G l^{2}} .
\end{aligned}
$$

Then the bulk action growth rate of a charged black hole in $f(R)$ gravity within the WDW patch in the late time approximation limit can be calculated:

$$
\begin{aligned}
\frac{\mathrm{d} \mathcal{A}_{\mathrm{bk}}}{\mathrm{d} t}= & \frac{\left(r_{-}^{3}-r_{+}^{3}\right)\left(1+f^{\prime}\left(R_{0}\right)\right)}{2 G l^{2}} \\
& +\frac{1}{2} q^{2}\left(\frac{1}{r_{-}}-\frac{1}{r_{+}}\right) .
\end{aligned}
$$

And the contribution from the $\mathrm{YGH}$ surface term is

$$
\begin{aligned}
\frac{\mathrm{d} \mathcal{A}_{\mathrm{bd}}}{\mathrm{d} t}= & \left(1+f^{\prime}\left(R_{0}\right)\right)\left(r_{+}-r_{-}\right) \\
& \times \frac{2 l^{2}+3\left(r_{-}^{2}+r_{+} r_{-}+r_{+}^{2}\right)}{2 G l^{2}} \\
& -\frac{q^{2}\left(r_{+}-r_{-}\right)}{2 r_{-} r_{+}} .
\end{aligned}
$$

Then we can get the total action growth rate,

$$
\begin{aligned}
\frac{\mathrm{d} \mathcal{A}}{\mathrm{d} t} & =\frac{\mathrm{d} \mathcal{A}_{\mathrm{bk}}}{\mathrm{d} t}+\frac{\mathrm{d} \mathcal{A}_{\mathrm{bd}}}{\mathrm{d} t} \\
& =\frac{\left(r_{+}-r_{-}\right)\left(1+f^{\prime}\left(R_{0}\right)\right)\left(l^{2}+r_{-}^{2}+r_{+}^{2}+r_{-} r_{+}\right)}{G l^{2}} \\
& =\mu_{-} Q-\mu_{+} Q,
\end{aligned}
$$

where the chemical potentials at the inner and outer horizon are $\mu_{ \pm}=\frac{q \sqrt{1+f^{\prime}\left(R_{0}\right)}}{r_{+}}$. Clearly, the action growth rate of the static charged black holes in $f(R)$ gravity can also saturate Eq. (4). It means that the higher order derivative of the curvature term does not reduce the action growth rate of black holes within the WDW patch in the late time approximation limit. Though the contributions from the null surfaces and the joints were considered in Ref. [23], the result is the same as ours.
4.3 Action growth rate of Kerr-Newman black holes in $f(R)$ gravity

The KN solution for a constant curvature black hole in $f(R)$ gravity was found in Ref. [58]:

$$
\begin{aligned}
\mathrm{d} s^{2}= & -\left(\frac{\triangle_{r}}{\rho^{2} \Xi^{2}}-\frac{\triangle_{\theta} \sin ^{2} \theta}{\rho^{2} \Xi^{2}} a^{2}\right) \mathrm{d} t^{2} \\
& +2 \frac{a \triangle_{r} \sin ^{2} \theta-a\left(r^{2}+a^{2}\right) \triangle_{\theta} \sin ^{2} \theta}{\rho^{2} \Xi^{2}} \mathrm{~d} t \mathrm{~d} \phi \\
& +\frac{\rho^{2}}{\triangle_{r}} \mathrm{~d} r^{2}+\frac{\rho^{2}}{\triangle_{\theta}} \mathrm{d} \theta^{2} \\
& +\frac{\sin ^{2} \theta}{\rho^{2} \Xi^{2}}\left[\left(r^{2}+a^{2}\right)^{2} \triangle_{\theta}-a^{2} \triangle_{r} \sin ^{2} \theta\right] \mathrm{d} \phi^{2},
\end{aligned}
$$

where $a$ is the rotating parameter and

$$
\begin{aligned}
\triangle_{r} & =\left(a^{2}+r^{2}\right)\left(1-\frac{R_{0}}{12} r^{2}\right)+\frac{G q^{2}}{1+f^{\prime}\left(R_{0}\right)}-2 G m r \\
\triangle_{\theta} & =1+\frac{R_{0}}{12} a^{2} \cos ^{2} \theta \\
\Xi & =1+\frac{R_{0}}{12} a^{2} \\
\rho^{2} & =r^{2}+a^{2} \cos ^{2} \theta
\end{aligned}
$$

Here we take the value of the Ricci scalar $R_{0}$ as a constant which is the same as Eq. (52). We can solve the mass parameter $m$ and electric charge parameter $q$ from $\triangle_{r}\left(r_{ \pm}\right)=0$,

$$
\begin{aligned}
m= & \frac{\left(r_{-}+r_{+}\right)\left(a^{2}+l^{2}+r_{-}^{2}+r_{+}^{2}\right)}{2 G l^{2}}, \\
q^{2}= & \frac{\left(1+f^{\prime}\left(R_{0}\right)\right)}{G l^{2}}\left(a^{2}\left(r_{-} r_{+}-l^{2}\right)\right. \\
& \left.+r_{-} r_{+}\left(l^{2}+r_{-}^{2}+r_{-} r_{+}+r_{+}^{2}\right)\right) .
\end{aligned}
$$

The electromagnetic field is described by

$$
\begin{aligned}
A= & -\frac{q r}{\rho^{2}}\left(\frac{\mathrm{d} t}{\Xi}-a \sin ^{2} \theta \frac{\mathrm{d} \phi}{\Xi}\right) \\
F= & -\frac{q\left(r^{2}-a^{2} \cos ^{2} \theta\right)}{\rho^{4}}\left(\frac{\mathrm{d} t}{\Xi}-a \sin ^{2} \theta \frac{\mathrm{d} \phi}{\Xi}\right) \wedge \mathrm{d} r \\
& -\frac{2 q r a \cos \theta \sin \theta}{\rho^{4}} \mathrm{~d} \theta \wedge\left(a \frac{\mathrm{d} t}{\Xi}-\left(r^{2}+a^{2}\right) \frac{\mathrm{d} \phi}{\Xi}\right) .
\end{aligned}
$$

The action growth rate of the bulk part can be calculated:

$$
\begin{aligned}
\frac{\mathrm{d} \mathcal{A}_{b k}}{\mathrm{~d} t}= & \left(1+f^{\prime}\left(R_{0}\right)\right) l^{2}\left(r_{-}-r_{+}\right) \frac{a^{2}+r_{-}^{2}+r_{+}^{2}+r_{-} r_{+}}{2 G\left(l^{2}-a^{2}\right)^{2}} \\
& -\frac{l^{4} q^{2}\left(r_{+}-r_{-}\right)\left(a^{2}-r_{-} r_{+}\right)}{2\left(l^{2}-a^{2}\right)^{2}\left(a^{2}+r_{-}^{2}\right)\left(a^{2}+r_{+}^{2}\right)} .
\end{aligned}
$$


For the boundary term, we take the same procedure as in $G R$. Using the condition $\triangle_{r}\left(r_{ \pm}\right)=0$, we have

$$
\begin{aligned}
\frac{\mathrm{d} \mathcal{A}_{b d}}{\mathrm{~d} t}= & \frac{\left(1+f^{\prime}\left(R_{0}\right)\right) l^{2}\left(r_{+}-r_{-}\right)}{2 G\left(l^{2}-a^{2}\right)^{2}} \\
& \times\left(a^{2}+l^{2}+2\left(r_{-}^{2}+r_{+} r_{-}+r_{+}^{2}\right)\right) .
\end{aligned}
$$

The angular velocity $\Omega$, electric potential $\mu$, physical mass $M$, charge $Q$, and angular momentum $J$ are [58]

$$
\begin{aligned}
\Omega_{ \pm} & =\frac{a}{a^{2}+r_{ \pm}^{2}}, \\
\mu_{ \pm} & =\frac{q r_{ \pm}}{\Xi\left(a^{2}+r_{ \pm}^{2}\right)}, \\
M & =\left(1+f^{\prime}\left(R_{0}\right)\right) \frac{m}{\Xi}, \\
Q & =\frac{q}{\Xi}, \\
J & =\left(1+f^{\prime}\left(R_{0}\right)\right) \frac{a m}{\Xi^{2}} .
\end{aligned}
$$

With the help of these quantities, we finally obtain

$$
\frac{\mathrm{d} \mathcal{A}}{\mathrm{d} t}=\left(M-\Omega_{+} J-\mu_{+} Q\right)-\left(M-\Omega_{-} J-\mu_{-} Q\right) .
$$

So we confirmed the conjecture of Ref. [32].

In the limit $a \rightarrow 0$, the KN black hole recovers to the charged black hole which can be seen from the metrics (6) and (64). The angular velocity and angular momentum will also approach zero. Therefore, the action growth rate can recover to the charged black hole case. Furthermore, in the neutral limit, $Q \rightarrow 0$, the inner horizon will disappear, i.e., $r_{-} \rightarrow 0$. In this case, the term $\mu_{+} Q$ approaches zero and the term $\mu_{-} Q$ approaches $2 M$, so the action growth rate approaches $2 M$, which recovers to the neutral case. These analyses are valid not only for GR but also for massive gravity and $f(R)$ gravity.

What we have done shows that all the action growth rates of neutral, charged, and KN black holes in $f(R)$ gravity can saturate the conjecture (4), because what we have investigated is the case of constant curvature. From the action (48) we can see that the higher derivative term, i.e., the $f(R)$ term, only contributes to the cosmology constant for the constant curvature case. In other words, the higher derivative term has no contribution to the action growth rate. Though the boundary term is changed, the result still can saturate the bound (4). As for the other higher derivative cases, the results may be different, and this is an interesting question to investigate.

\section{Conclusion and discussion}

The action of a stationary AdS black hole within the WDW patch has been related to the quantum complexity of a holographic state. Following the procedure in Ref. [32], we calculated the action growth rate of the static and stationary AdS black holes in massive gravity and $f(R)$ gravity within the WDW patch at the late time approximation.

For massive gravity, we found that the action growth rate of the black hole does not depend on the parameter $k$. That is to say, no matter what the topology of the black hole is, the action growth rate will saturate the bound (4). The action growth rate equals $2 M$ for a neutral static AdS black hole, and it is the difference value of $M-\mu Q$ at the inner and outer horizons for a charged static AdS black hole. Though the structure of the growth rate does not change, the effect of the massive graviton terms will slow down the growth rate for the charged static AdS black holes in massive gravity which has the same mass and charge as the black holes in GR. But the massive graviton terms do not have effect on the neutral static AdS black holes. These results are consistent with that of Ref. [33].

For the $f(R)$ gravity, we found that the bound (4) of the action growth rate does not change for neutral, charged, and KN AdS black holes. Because the $f(R)$ term for the case of constant curvature only contributes to the action a cosmology constant term, so the results are expected. Our results checked the conjecture proposed in Ref. [32] and showed more abundant features on the action growth rate as well as the quantum complexity. For other higher derivative gravities, this conjecture should be checked in detail.

Though the topology of the spacetime studied in this paper has no influence on the action growth rate, the result may be different for other nontrivial topologically black hole spacetime. It is well known that there are many interesting effects of the nontrivial topology in black hole spacetime. For example, black holes with topologically nontrivial $S^{2}$-cycle have the same conserved charge at infinity as the Breckenridge-Myers-Peet-Vafa (BMPV) black hole but greater entropy than the BMPV black hole [66,67]. As the action of the WDW patch of the black hole is dual to the quantum complexity of the quantum state, to study the influence of the nontrivial topology on the action growth rate will be a further test on the CA duality. And this may lead to a deeper understanding of the black hole interior. However, the spacetime of the black hole with nontrivial topology found in Refs. [66,67] is asymptotically flat, and we have no idea of the dual quantum state of the flat spacetime, and hence of the quantum complexity. The solutions found in Ref. [66] can be generalized to six dimensions, and the spacetime is asymptotically $\mathrm{AdS}_{3} \times S^{3}$, however, the CFT dual of the bubbling geometry is still unknown [68,69]. Anyway, this would be an interesting direction.

Besides, the black hole interior has also been a mystery, we hope that we can learn more about this from the view point of quantum complexity. 
Acknowledgements This work was supported by the National Natural Science Foundation of China (Grants Nos. 11522541, 11375075, and 11675064), and the Fundamental Research Funds for the Central Universities (Grants Nos. lzujbky-2016-k04, lzujbky-2016-115, and lzujbky-2017-it69).

Open Access This article is distributed under the terms of the Creative Commons Attribution 4.0 International License (http://creativecomm ons.org/licenses/by/4.0/), which permits unrestricted use, distribution, and reproduction in any medium, provided you give appropriate credit to the original author(s) and the source, provide a link to the Creative Commons license, and indicate if changes were made. Funded by SCOAP ${ }^{3}$.

\section{References}

1. L. Susskind, Butterflies on the stretched horizon. arXiv:1311.7379

2. L. Susskind, Computational complexity and black hole horizons. Fortsch. Phys. 64, 24-43 (2016). arXiv:1403.5695

3. L. Susskind, Addendum to computational complexity and black hole horizons. Fortsch. Phys. 64, 44-48 (2016)

4. A.R. Brown, D.A. Roberts, L. Susskind, B. Swingle, Y. Zhao, Holographic complexity equals bulk action? Phys. Rev. Lett. 116, 191301 (2016). arXiv: 1509.07876

5. A.R. Brown, D.A. Roberts, L. Susskind, B. Swingle, Y. Zhao, Complexity, action, and black holes. Phys. Rev. D 93, 086006 (2016). arXiv: 1512.04993

6. P. Hosur, X.-L. Qi, D.A. Roberts, B. Yoshida, Chaos in quantum channels. JHEP 02, 004 (2016). arXiv:1511.04021

7. M. Alishahiha, Holographic Complexity. Phys. Rev. D 92, 126009 (2015). arXiv:1509.06614

8. L. Lehner, R.C. Myers, E. Poisson, R.D. Sorkin, Gravitational action with null boundaries. Phys. Rev. D 94, 084046 (2016). arXiv: 1609.00207

9. J.L.F. Barbon, E. Rabinovici, Holographic complexity and spacetime singularities. JHEP 01, 084 (2016). arXiv:1509.09291

10. D. Carmi, R.C. Myers, P. Rath, Comments on holographic complexity. JHEP 03, 118 (2017). arXiv: 1612.00433

11. S. Chapman, H. Marrochio, R.C. Myers, Complexity of formation in holography. JHEP 01, 062 (2017). arXiv: 1610.08063

12. O. Ben-Ami, D. Carmi, On volumes of subregions in holography and complexity. JHEP 11, 129 (2016). arXiv:1609.02514

13. A.R. Brown, L. Susskind, Y. Zhao, Quantum complexity and negative curvature. Phys. Rev. D 95, 045010 (2017). arXiv:1608.02612

14. D.A. Roberts, B. Yoshida, Chaos and complexity by design. JHEP 04, 121 (2017), arXiv:1610.04903

15. J. Couch, W. Fischler, P.H. Nguyen, Noether charge, black hole volume, and complexity, JHEP 03, 119 (2017). arXiv:1610.02038

16. J.L.F. Barbon, J. Martin-Garcia, Holographic complexity of cold hyperbolic black holes. JHEP 11, 181 (2015). arXiv:1510.00349

17. A. Reynolds, S.F. Ross, Divergences in holographic complexity. Class. Quant. Grav. 34, 105004 (2017). arXiv:1612.05439

18. R.-Q. Yang, Strong energy condition and the fastest computers. Phys. Rev. D 95, 086017 (2017). arXiv: 1610.05090

19. A. R. Brown, L. Susskind, The second law of quantum complexity. arXiv:1701.01107

20. H. Huang, X.-H. Feng, H. Lu, Holographic complexity and two identities of action growth. Phys. Lett. B 769, 357-361 (2017). arXiv:1611.02321

21. Y. Zhao, Complexity, boost symmetry, and firewalls. arXiv: 1702.03957

22. J. Tao, P. Wang, H. Yang, Testing holographic conjectures of complexity with Born-Infeld black holes. arXiv:1703.06297
23. P. Wang, H. Yang, S. Ying, Action growth in $f(R)$ gravity. Phys. Rev. D 96, 046007 (2017). arXiv: 1703.10006

24. R.-G. Cai, M. Sasaki, S.-J. Wang, Action growth of charged black holes with a single horizon. Phys. Rev. D 95, 124002 (2017). arXiv: 1702.06766

25. D. Momeni, M. Faizal, S. Bahamonde, R. Myrzakulov, Holographic complexity for time-dependent backgrounds. Phys. Lett. B 762, 276-282 (2016). arXiv: 1610.01542

26. N.S. Mazhari, D. Momeni, S. Bahamonde, M. Faizal, R. Myrzakulov, Holographic complexity and fidelity susceptibility as holographic information dual to different volumes in AdS. Phys. Lett. B 766, 94-101 (2017). arXiv:1609.00250

27. D. Momeni, M. Faizal, K. Myrzakulov, R. Myrzakulov, Fidelity susceptibility as holographic PV-criticality. Phys. Lett. B 765, 154158 (2017). arXiv: 1604.06909

28. D. Momeni, S.A.H. Mansoori, R. Myrzakulov, Holographic complexity in gauge/string superconductors. Phys. Lett. B 756, 354 357 (2016). arXiv:1601.03011

29. D. Momeni, M. Faizal, R. Myrzakulov, Holographic Cavalieri principle as a universal relation between holographic complexity and holographic entanglement entropy. arXiv:1703.01337

30. D. Stanford, L. Susskind, Complexity and shock wave geometries. Phys. Rev. D 90, 126007 (2014). arXiv:1406.2678

31. S. Lloyd, Ultimate physical limits to computation. Nature 406, 1047 (2000). arXiv:9908043

32. R.-G. Cai, S.-M. Ruan, S.-J. Wang, R.-Q. Yang, R.-H. Peng, Action growth for AdS black holes. JHEP 09, 161 (2016). arXiv: 1606.08307

33. W.-J. Pan, Y.-C. Huang, Holographic complexity and action growth in massive gravities. Phys. Rev. D 95, 126013 (2017). arXiv: 1612.03627

34. M. Alishahiha, A.F. Astaneh, A. Naseh, M.H. Vahidinia, On complexity for higher derivative gravities. JHEP 05, 009 (2017). arXiv: 1702.06796

35. B. Carter, Hamilton-Jacobi and Schrodinger separable solutions of Einstein's equations. Commun. Math. Phys. 10, 280 (1968)

36. J.F. Plebanski, M. Demianski, Rotating, charged, and uniformly accelerating mass in general relativity. Ann. Phys. 98, 98-127 (1976)

37. M.M. Caldarelli, G. Cognola, D. Klemm, Thermodynamics of Kerr-Newman-AdS black holes and conformal field theories. Class. Quant. Grav. 17, 399-420 (2000). arXiv:hep-th/9908022

38. C. de Rham, G. Gabadadze, Generalization of the Fierz-Pauli Action. Phys. Rev. D 82, 044020 (2010). arXiv: 1007.0443

39. C. de Rham, G. Gabadadze, A.J. Tolley, Resummation of massive gravity. Phys. Rev. Lett. 106, 231101 (2011). arXiv:1011.1232

40. K. Hinterbichler, Theoretical aspects of massive gravity. Rev. Mod. Phys. 84, 671-710 (2012). arXiv:1105.3735

41. S.F. Hassan, R.A. Rosen, Resolving the ghost problem in nonlinear massive gravity. Phys. Rev. Lett. 108, 041101 (2012). arXiv: 1106.3344

42. S.F. Hassan, R.A. Rosen, A. Schmidt-May, Ghost-free massive gravity with a general reference metric. JHEP 02, 026 (2012). arXiv: 1109.3230

43. D. Vegh, Holography without translational symmetry. arXiv: 1301.0537

44. R.-G. Cai, Y.-P. Hu, Q.-Y. Pan, Y.-L. Zhang, Thermodynamics of black holes in massive gravity. Phys. Rev. D 91, 024032 (2015). arXiv: 1409.2369

45. J. Xu, L.-M. Cao, Y.-P. Hu, P-V criticality in the extended phase space of black holes in massive gravity. Phys. Rev. D 91, 124033 (2015). arXiv: 1506.03578

46. S. Hendi, R. Mann, S. Panahiyan, B.Eslam Panah, Van der Waals like behavior of topological AdS black holes in massive gravity. Phys. Rev. D 95, 021501 (2017). arXiv:1702.00432 
47. S.H. Hendi, G.-Q. Li, J.-X. Mo, S. Panahiyan, B.Eslam Panah, New perspective for black hole thermodynamics in Gauss-BonnetBorn-Infeld massive gravity. Eur. Phys. J. C 76, 571 (2016). arXiv: 1608.03148

48. S .H. Hendi, B Eslam Panah, S. Panahiyan, Einstein-Born-Infeldmassive gravity: AdS-black hole solutions and their thermodynamical properties. JHEP 11, 157 (2015). arXiv:1508.01311

49. S .H. Hendi, S. Panahiyan, B.Eslam Panah, M. Momennia, Phase transition of charged black holes in massive gravity through new methods. Ann. Phys. 528, 819-834 (2016). arXiv:1506.07262

50. Y.-F. Cai, D.A. Easson, C. Gao, E.N. Saridakis, Charged black holes in nonlinear massive gravity. Phys. Rev. D 87, 064001 (2013). arXiv:1211.0563

51. D.-C. Zou, R.-H. Yue, M. Zhang, Reentrant phase transitions of higher-dimensional AdS black holes in dRGT massive gravity. Eur. Phys. J. C 77, 256 (2017). arXiv:1612.08056

52. D.-C. Zou, Y.-Q. Liu, R.-H. Yue, Behavior of Quasinormal Modes and Van der Waals like phase transition of charged AdS black holes in massive gravity. Eur. Phys. J. C 77, 365 (2017). arXiv: 1702.08118

53. A. Guarnizo, L. Castaneda, J.M. Tejeiro, Boundary term in metric $f(R)$ gravity: field equations in the metric formalism. Gen. Rel. Grav. 42, 2713-2728 (2010). arXiv:1002.0617

54. T. Moon, Y.S. Myung, E.J. Son, $\mathrm{f}(\mathrm{R})$ black holes. Gen. Rel. Grav. 43, 3079-3098 (2011). arXiv:1101.1153

55. A. Sheykhi, Higher-dimensional charged $f(R)$ black holes. Phys. Rev. D 86, 024013 (2012). arXiv: 1209.2960

56. S. Chen, X. Liu, C. Liu, J. Jing, $P-V$ criticality of AdS black hole in $f(R)$ gravity. Chin. Phys. Lett. 30, 060401 (2013). arXiv: 1301.3234

57. A. de la Cruz-Dombriz, D. Saez-Gomez, Black holes, cosmological solutions, future singularities, and their thermodynamical properties in modified gravity theories. Entropy 14, 1717-1770 (2012). arXiv:1207.2663
58. J.A.R. Cembranos, A. de la Cruz-Dombriz, P.Jimeno Romero, Kerr-Newman black holes in $f(R)$ theories. Int. J. Geom. Meth. Mod. Phys. 11, 1450001 (2014). arXiv: 1109.4519

59. J. Wang, W. Xu, X.-H. Meng, Entropy relations of black holes with multihorizons in higher dimensions. Phys. Rev. D 89, 044034 (2014). arXiv:1312.3057

60. S. Chakraborty, S. SenGupta, Spherically symmetric brane spacetime with bulk $f(\mathcal{R})$ gravity. Eur. Phys. J. C 75, 11 (2015). arXiv: 1409.4115

61. S. Chakraborty, S. SenGupta, Solving higher curvature gravity theories. Eur. Phys. J. C 76, 552 (2016). arXiv:1604.05301

62. S.H. Hendi, B.Eslam Panah, R. Saffari, Exact solutions of threedimensional black holes: Einstein gravity versus $F(R)$ gravity. Int. J. Mod. Phys. D 23, 1450088 (2014). arXiv: 1408.5570

63. S.H. Hendi, B. Eslam Panah, S. Panahiyan, M. Momennia, F(R) gravity's rainbow and its Einstein counterpart. Adv. High Energy Phys. 2016, 9813582 (2016). arXiv:1607.03383

64. Y.-F. Cai, E.N. Saridakis, Cosmology of $F(R)$ nonlinear massive gravity. Phys. Rev. D 90, 063528 (2014). arXiv:1401.4418

65. Y.-F. Cai, F. Duplessis, E.N. Saridakis, $F(R)$ nonlinear massive theories of gravity and their cosmological implications. Phys. Rev. D 90, 064051 (2014). arXiv:1307.7150

66. G.T. Horowitz, H.K. Kunduri, J. Lucietti, Comments on black holes in bubbling spacetimes. JHEP 06, 048 (2017). arXiv:1704.04071

67. H.K. Kunduri, J. Lucietti, Black hole non-uniqueness via spacetime topology in five dimensions. JHEP 10, 082 (2014). arXiv: 1407.8002

68. K. Skenderis, M. Taylor, The fuzzball proposal for black holes. Phys. Rep. 467, 117 (2008). arXiv:0804.0552

69. S. Giusto, E. Moscato, R. Russo, $\mathrm{AdS}_{3}$ holography for $1 / 4$ and $1 / 8$ BPS geometries. JHEP 11, 004 (2015). arXiv:1507.00945 